\title{
Structural, electronic and magnetic properties of Ti-doped polar and nonpolar GaN surfaces
}

Mendoza Estrada, Victor Julio; González García, Alvaro; López Pérez, William; Pinilla, Carlos C. ; González Hernández, Rafael J.

\begin{abstract}
Based on density functional theory, first-principles calculations were performed in order to study the titanium incorporation on polar and nonpolar GaN surfaces. The formation energy calculations indicate that Ti impurity atoms prefer to incorporate in surface layers (first and second) of GaN. It is also concluded that the incorporation of $\mathrm{Ti}$ atoms in Ga-substitutional sites are more energetically favorable compared with $\mathrm{N}$-substitutional or interstitial sites on the polar and nonpolar GaN surfaces. For Tirich growth conditions, formation energy calculations show the formation of $\mathrm{Tix}_{\mathrm{x}} \mathrm{N}$ layers on the a and $c$ GaN surfaces, which corroborates recent experimental observations. Results also display that the $3 \mathrm{~d}$-Ti states are the responsible for the metallization of the surface on the $\mathrm{c}$ and $\mathrm{m}$ planes, forming an intermetallic alloy $(\mathrm{Tix} N)$, which could be used as low-resistance ohmic contacts for GaN. In addition, the magnetic properties with Ti doping show magnetization of about $1.0 \mu_{\mathrm{B}} / \mathrm{Ti}$ atom for the nonpolar GaN surfaces.
\end{abstract}

\section{Keywords}

Magnetic materials, Nitrides, Ohmic contacts, Semiconducting III-V materials. 